\title{
Angle-resolved photoemission observation of the superconducting-gap minimum and its relation to the nesting vector in the phonon-mediated superconductor $\mathrm{YNi}_{2} \mathrm{~B}_{2} \mathrm{C}$
}

\author{
T. Baba, ${ }^{1, *}$ T. Yokoya, ${ }^{1,2, \dagger}$ S. Tsuda, ${ }^{1, \$}$ T. Watanabe,,${ }^{1, \S}$ M. Nohara, ${ }^{3, \|}$ H. Takagi, ${ }^{3}$ T. Oguchi, ${ }^{4}$ and S. Shin ${ }^{1,5}$ \\ ${ }^{1}$ Institute for Solid State Physics, University of Tokyo, 5-1-5 Kashiwanoha, Kashiwa, Chiba 277-8581, Japan \\ ${ }^{2}$ The Graduate School of Natural Science and Technology, Okayama University, 3-1-1 Tsushima-naka, Okayama 700-8530, Japan \\ ${ }^{3}$ Department of Advanced Materials Science, University of Tokyo, 5-1-5 Kashiwanoha, Kashiwa, Chiba 277-8565, Japan \\ ${ }^{4}$ Department of Quantum Matter, Graduate school of Advanced Sciences of Matter (ADSM), Hiroshima University, 1-3-1 Kagamiyama, \\ Higashi-Hiroshima 739-8526, Japan \\ ${ }^{5}$ RIKEN SPring-8 Center, 1-1-1 Koto, Sayo-chou, Sayo-gun, Hyogo 679-5143, Japan
}

(Received 12 March 2010; published 17 May 2010)

\begin{abstract}
We have performed ultrahigh-resolution angle-resolved photoemission spectroscopy to directly study the large superconducting (SC) gap anisotropy of $\mathrm{YNi}_{2} \mathrm{~B}_{2} \mathrm{C}$. We succeed in measuring momentum $(k)$ dependence of SC gap for individual Fermi surface (FS) sheets, which demonstrates complexity of SC gap in a phononmediated superconductor. Within measured $k$ regions on FS sheets, we find a pointlike minimum of SC gap, whose $k$ positions can be connected by the known nesting vector. This shows close correlation between the nesting vector and node formation.
\end{abstract}

DOI: 10.1103/PhysRevB.81.180509

PACS number(s): 74.25.Jb, 74.70.Dd, 79.60.-i

Superconductivity is a macroscopically emerged quantum phenomenon derived from many body interactions inside a solid. Magnitude of a superconducting (SC) energy gap is a fundamental parameter and its momentum $(k)$ dependence reflects symmetry of the pairing wave function of a Cooper pair as well as interactions of electrons with elementary excitations. In a conventional phonon-mediated superconductor, a simple $s$-wave paring wave function leads to an isotropic SC gap, with slight deviation due to $k$-dependent electronic states and/or electron-phonon interaction. ${ }^{1}$ Large superconducting gap anisotropy with nodes (zero gap regions) realizes in superconductors with strong electron correlation (high- $T_{\mathrm{c}}$ cuprates, heavy fermions, ruthenate, and organic materials) and is due to the change of a sign in the pairing wave functions. ${ }^{2}$ In such materials, the pairing mechanisms other than phonon are actively discussed. On the other hand, regarded as phonon-mediated superconductors, ${ }^{3-7}$ borocarbide $\mathrm{YNi}_{2} \mathrm{~B}_{2} \mathrm{C}$ and $\mathrm{LuNi}_{2} \mathrm{~B}_{2} \mathrm{C}$ have turned out to have large $\mathrm{SC}$ gap anisotropy with nodes. ${ }^{8-12}$ Since such an extremely large gap anisotropy cannot be obtained from a simple $s$-wave paring wave function that is assumed for a phonon-mediated superconductor, it is surprising and a challenge of condensed matter physics ${ }^{13}$ as to what kind of interactions play an essential role and how such an extremely large anisotropy is produced.

For understanding the origin of the large SC gap anisotropy, it has been considered essential to determine the direction and type of nodal structure and to study its relation to nesting vector ${ }^{14,15}$ reported in borocarbides. However, results are controversial: the magnetic field orientation dependence of thermal conductivity, ${ }^{16}$ ultrasonic attenuation, ${ }^{17}$ and scanning tunneling microscopy/spectroscopy in the vortex state ${ }^{18}$ have reported point nodes located along [100] and [010] directions while field-angle-dependent heat capacity suggested a linelike nodal structure. ${ }^{19}$ Even, isotropic two-gap superconductivity has been proposed by specific heat ${ }^{20}$ and directional point-contact spectroscopy measurements. ${ }^{21}$ This situation is mainly because that the informations obtained from these techniques are indirect and neither give the shape of Fermi surface (FS) nor the $k$ position of the node on FS. Therefore, experimental study of $k$ dependence of SC gap in detail that determines the type of nodal structure (point or line type) and location of the nodes on FS sheets is highly desired. For this purpose, high-resolution angle-resolved photoemission spectroscopy (ARPES) is a powerful experimental technique that can directly observe temperature (T)-dependent $k$-resolved electronic structure of solids. But, previous ARPES did not report systematic $k$-dependent superconducting gap. ${ }^{22,23}$

In this Rapid Communication, we report ARPES of (001) single crystal $\mathrm{YNi}_{2} \mathrm{~B}_{2} \mathrm{C}$. We succeed in measuring $k$ dependence of SC gap for individual FS sheets. The results show that the magnitude and anisotropy of SC gap exhibit FS sheet dependence, which demonstrates importance of $k$-resolved electronic structure study for borocarbide superconductors. We also find that a pointlike minimum of SC gap is located on a cylindrical FS sheet around X-P and are found to be connected by the known nesting vector. This result indicates that nested FS plays a crucial role for the node formation.

Single crystals of $\mathrm{YNi}_{2} \mathrm{~B}_{2} \mathrm{C}$ were grown by a floatingzone method. ${ }^{24} \mathrm{SC}$ transition $T\left(T_{\mathrm{c}}\right)$ of $15.4 \mathrm{~K}$ was determined by the onset of magnetic susceptibility measurements. The residual resistivity ratio was 37.4. Sample orientations were measured ex situ by using Laue backscattering and further confirmed in situ by the symmetry of ARPES spectra. All the ARPES data presented here have been measured with a high-resolution hemispherical analyzer using the monochromatic He $I \alpha$ resonance line $(21.218 \mathrm{eV})$. Energy resolutions for FS mapping and SC-gap measurements were set to $\sim 30 \mathrm{meV}$ and $1.7 \mathrm{meV}$, respectively. The angular resolution was set to $0.18^{\circ}$, corresponding to $k$ resolution of $\pm 0.0067 \AA^{-1}$. The sample $T$ for the FS mapping was $20 \mathrm{~K}$, while those of SC-gap measurements were $6 \mathrm{~K}$ (SC state) and $18 \mathrm{~K}$ (normal state), respectively. The base pressure of our spectrometer was better than $2 \times 10^{-11}$ Torr. Singlecrystal clean surfaces of $\mathrm{YNi}_{2} \mathrm{~B}_{2} \mathrm{C}(001)$ were prepared in situ 

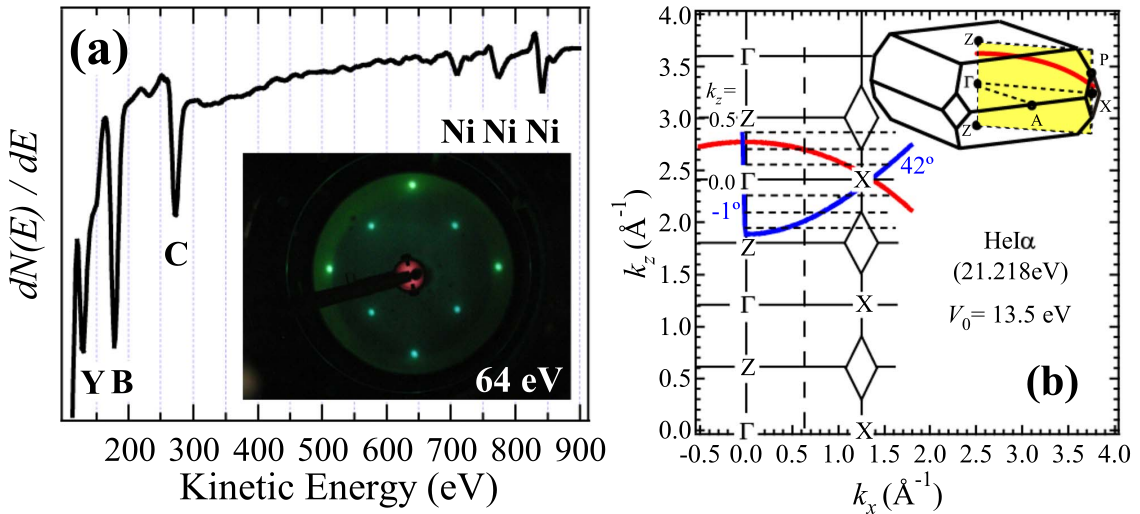

FIG. 1. (Color online) (a) First derivative Auger spectrum of $\mathrm{YNi}_{2} \mathrm{~B}_{2} \mathrm{C}(001)$. Inset: the LEED pattern of $\mathrm{YNi}_{2} \mathrm{~B}_{2} \mathrm{C}(001)-1 \times 1$ at $64 \mathrm{eV}$ electron-beam energy. (b) Typical spherical path through the $\mathrm{BZ}$ at $21.218 \mathrm{eV}(\mathrm{He} I \alpha)$ for states at $E_{\mathrm{F}}$. Upper right corner: $\mathrm{BZ}$ and the $\Gamma$-X-P-Z plane.

by repeating Ar-ion bombardment for several hours and flash heating at $>1200{ }^{\circ} \mathrm{C}$. No traces of oxygen, sulfur nor other impurities were detected in the Auger electron spectra [Fig. $1(\mathrm{a})]$, and a clear $(1 \times 1)$ low-energy electron-diffraction (LEED) pattern [inset of Fig. 1(a)] having the same symmetry as the bulk crystal was observed. The Fermi level $\left(E_{\mathrm{F}}\right)$ of samples was referenced to that of a gold film evaporated onto the sample substrate and its accuracy was estimated to be better than $5 \mathrm{meV}$ for the FS mapping and $0.2 \mathrm{meV}$ for $\mathrm{SC}$-gap measurements. The band calculations of $\mathrm{YNi}_{2} \mathrm{~B}_{2} \mathrm{C}$ were performed by a full potential linearized augmented plane wave (FLAPW) method for detailed comparison with FS sheets observed experimentally. ARPES defines the $k_{\|}$ component of an electron. For three-dimensional electronic structures of $\mathrm{YNi}_{2} \mathrm{~B}_{2} \mathrm{C}$, perpendicular component $k_{\perp}$ can be obtained by assuming a free-electron final state model with an inner potential $V_{0}$. Here we used $V_{0}=13.5 \mathrm{eV}$ by comparing observed FS sheets with calculated ones.

Figure 2(a) is an ARPES intensity map obtained from a plot of spectral intensity $( \pm 12.5 \mathrm{meV}$ window centered at

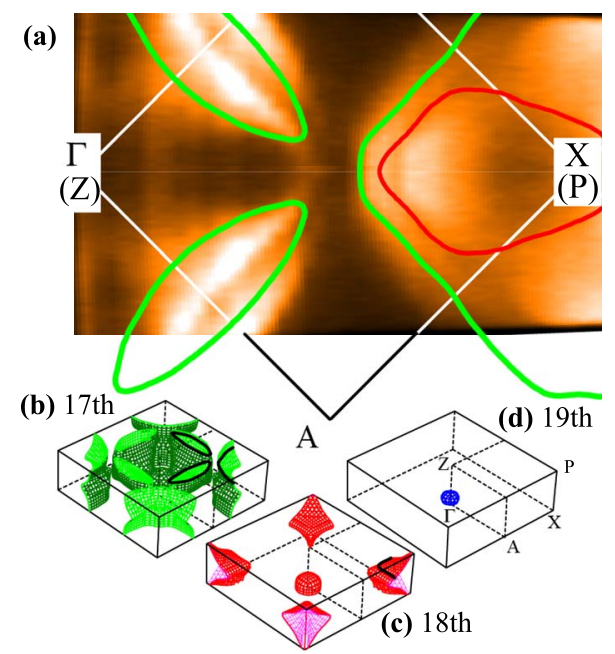

FIG. 2. (Color online) (a) The ARPES intensity map at $E_{\mathrm{F}}$ (energy window $=E_{\mathrm{F}} \pm 12.5 \mathrm{meV}$ ) of $\mathrm{YNi}_{2} \mathrm{~B}_{2} \mathrm{C}(001)$, along with the calculated FSs. [(b)-(d)] Calculated FSs from 17th, 18th, and 19 th bands, respectively.
$\left.E_{\mathrm{F}}\right)$ over the $\mathrm{BZ}$, where white regions correspond to $\mathrm{FS}$ sheets. We observed two high intensity lines perpendicular to the $\Gamma(\mathrm{Z})$-A line. We also found that a high-intensity region around the $\mathrm{X}(\mathrm{P})$ point that can be divided into two squarelike sheets centered at the $\mathrm{X}(\mathrm{P})$ point by a lower intensity line (see also Fig. 4). In Figs. 2(b)-2(d), we also show three FS sheets (from 17th, 18th, and 19th bands, respectively) calculated by the FLAPW method. To correlate observed intensity variation with calculated FS sheets, we superimposed intersections of calculated FS sheets with a measured $k$ plane upon experimental FSs. The two high-intensity lines perpendicular to $\Gamma(\mathrm{Z})$-A can be ascribed to the two sides of an elliptic intersection of the FS sheet of 17th band. The two components of the higher-intensity region around $\mathrm{X}(\mathrm{P})$ can be ascribed to FS sheets of 17 th and 18th bands.

The observed intensity variation at $E_{\mathrm{F}}$ being able to be correlated with calculated FS sheets, we have performed low- $T$ ultrahigh-resolution ARPES for SC and normal states in steps of $1^{\circ}$ for all points of first BZ to investigate the $k$ dependence of SC gaps. Figure 3(a) shows regions of FS sheets for which we measured SC gap values and Fig. 3(b) shows ARPES spectra for selected points on FS sheets. We find that all ARPES spectra (including spectra not shown in this manuscript) exhibit a remarkable $T$ dependence, indicative of opening of a SC gap below $T_{\mathrm{c}}$. Sizable SC gap opening at every measured $k$ point of first BZ suggests absence of a line node along the $k_{\mathrm{z}}$ direction within measured FS sheets.

To investigate the $k$ dependence of magnitude of SC gaps $(\Delta)$, we numerically fitted the data with the modified BCS function, as described previously for the $\mathrm{MgB}_{2} \cdot{ }^{25}$ The modified BCS function was first multiplied with a Fermi-Dirac (FD) function of the measured $T$ and then convoluted with a Gaussian with a full width at half maximum equal to the experimental energy resolution. $\Delta$ values for all measured points along FSs are plotted in Fig. 3(c). One can observe larger SC gap values of the elliptic intersection of the 17 th FS sheet $\left(2 \Delta / k_{\mathrm{B}} T_{\mathrm{c}}=3.5-4.8\right)$ than those of $\mathrm{X}(\mathrm{P})$-centered 17 th $\left(2 \Delta / k_{\mathrm{B}} T_{\mathrm{c}}=2.3-3.2\right)$ and 18 th $\left(2 \Delta / k_{\mathrm{B}} T_{\mathrm{c}}=2.7-3.2\right)$ FS sheets. As for the $k$ dependence, the SC gap values from the elliptic FS sheet show marked $k$ dependence with minimum along the $\Gamma(\mathrm{Z})$-A direction and smooth increase away from it with a rather flat bottom $\left(0.3-0.4 \mathrm{~A}^{-1}\right)$. Contrary, the SC 

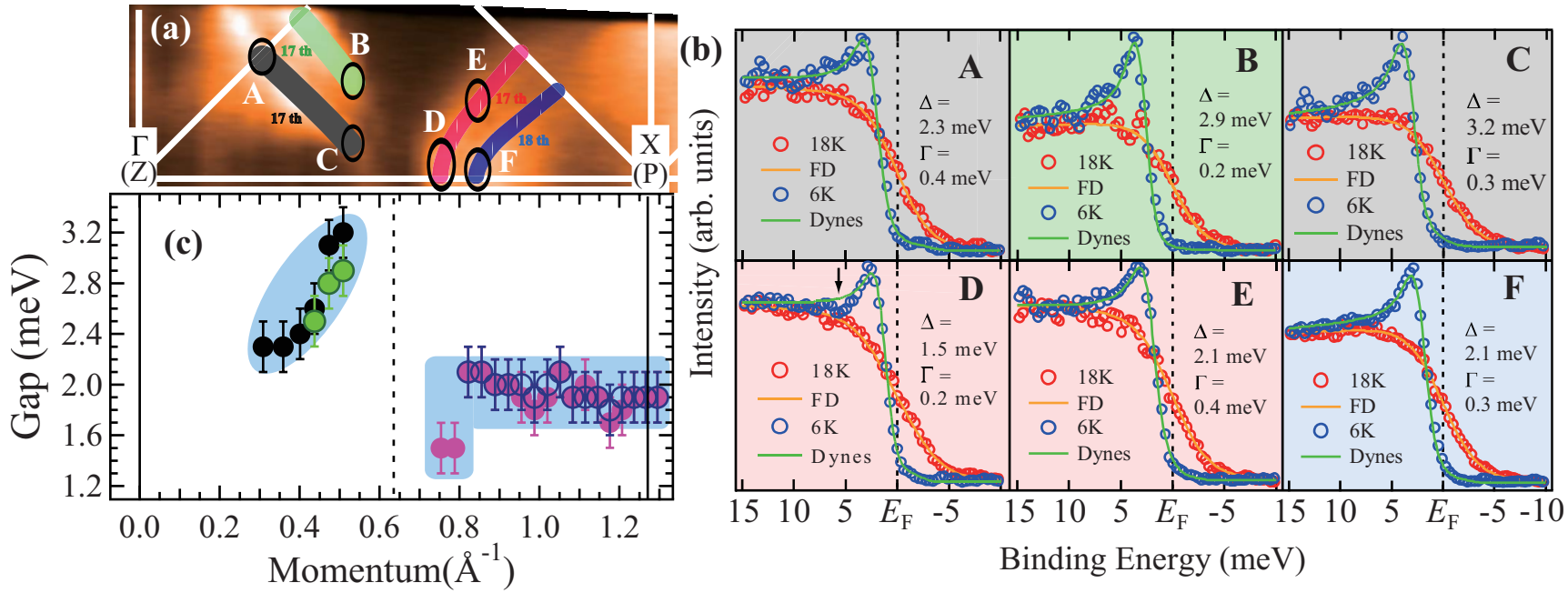

FIG. 3. (Color online) (a) Regions of FSs for which we measured SC gaps. (b) The selected low-T high-resolution ARPES spectra. Blue (dark) and red (light) circles represent the experimental results measured at $6 \mathrm{~K}$ (SC) and $18 \mathrm{~K}$ (normal), respectively. Green and yellow lines (on the SC and normal state spectra) represent the fitting results by using the modified BCS function and the FD function, respectively. (c) A plot of SC gap values on those FSs, where the color (tone) of symbols corresponds to the color (tone) of measured $k$ regions shown in Fig. 3(a).

gap values along $\mathrm{X}(\mathrm{P})$-centered 17 th and 18th FS sheets are found nearly isotropic but with a pointlike minimum along the $\Gamma(\mathrm{Z})-\mathrm{X}(\mathrm{P})$ direction only on the 17 th FS sheet. This pointlike minimum has the lowest $\mathrm{SC}$ gap value of $1.5 \mathrm{meV}$ $\left(2 \Delta / k_{\mathrm{B}} T_{\mathrm{c}}=2.3\right)$ within the measured $k$ plane. We attribute the difference of the anisotropic gap ratio $\left(\Delta_{\max } / \Delta_{\min }\right.$, where $\Delta_{\max }$ and $\Delta_{\min }$ are the maximum and minimum SC gap values, respectively) between the present study $(3.2 / 1.5=2.1)$ and other transport measurements (10-100) (Refs. 9 and 16) to the limitation of accessible $k$ regions from our experimental condition. The observed anisotropy of SC gap does not agree with an isotropic two-gaps suggested by Refs. 20 and 21 . The result also shows that the nodal structure is not a line node at least on 17 th and $\mathrm{X}(\mathrm{P})$-centered 18 th FSs, which are expected to have dominant contribution to the total density of state and therefore to the SC gap anisotropy observed from other experiments. In addition, we find that only the superconducting spectrum at D point where pointlike SC gap minimum is observed has a peak-dip-hump structure [an arrow in Fig. 3(b) for the dip] indicative of strong coupling, which indicates $k$ dependence of electron-phonon coupling.

In borocarbide superconductors, it is suggested that the FS has a nesting feature whose nesting vector is $Q$ $\sim(0.55,0,0)$ from band calculations, ${ }^{14}$ which was experimentally suggested by two-dimensional angular correlation of electron-positron annihilation. ${ }^{15}$ In addition, from inelastic neutron-scattering measurements, ${ }^{26}$ Kohn anomalies have been observed with a gradual phonon softening at wave vector close to $Q \sim(0.55,0,0)$. Moreover, from nuclear magnetic resonance study in the normal state, ${ }^{27,28} 1 / T_{1} T$ of ${ }^{11} \mathrm{~B}$ increases monotonously as $T$ decreases though the Knight shift which correspond to the susceptibility at $q=0$ weakly depends on $T$. The results suggested an enhancement of antiferromagnetic fluctuations at low $T$, which are expected to originate from the nesting of the FSs because the generalized susceptibility from band calculations shows a peak at $q \sim Q .{ }^{14}$ In order to understand the relationship between the pointlike SC gap minimum and the nesting vector, we plotted the ARPES intensity map over the first BZ by using the fourfold symmetry of the crystal, as shown in Fig. 4. Two pointlike SC gap minima are connected by vectors I. An estimated magnitude of the wave vector is $0.60 \pm 0.03$, which has the same direction and nearly the same magnitude as the nesting vector from previous works. ${ }^{14,15}$ More importantly, location of the vector I on the observed FSs is also in excellent agreement with the previous works. ${ }^{14,15}$ A slight enlargement of the magnitude may be due to the difference in $k_{\mathrm{z}}\left(k_{\mathrm{z}} \sim 0.25\right.$ of the vector I but $k_{\mathrm{z}}=0$ of the known nesting vector), which is consistent with the observation that the experimental FS sheets do not show a "crater structure" that is expected to be located around $k_{\mathrm{z}}=0.0$ from band calculations. ${ }^{14}$ On the other hand, we found that two SC gap minima on the elliptic FS sheet can be connected (vector II). Though it has the same direction as the known nesting vector with the magnitude of $0.56 \pm 0.04$ similar to that of the nesting vector, its location on the FS is different from the previous works. ${ }^{14,15}$

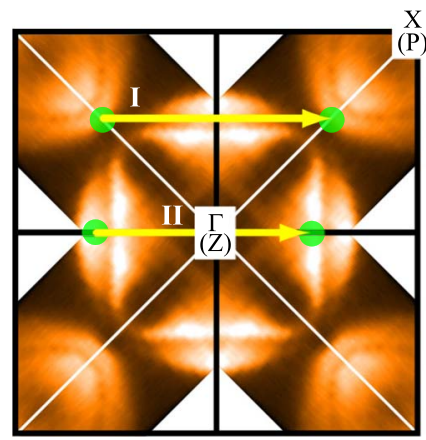

FIG. 4. (Color online) Symmetrized FS. The arrow I and II represent the vector connected to the pointlike SC gap minima on $\Gamma(\mathrm{Z})$-centered 17 th FS and local gap minima on $\mathrm{X}(\mathrm{P})$-centered 17 th FS, respectively. 
More recently, a quasiclassical Eilenberger theory taking into the two-dimensional FS calculated by local density approximation reported that point nodes are located at parts on FS connected by the known nesting vector at $k_{\mathrm{z}}=0.0 .^{29}$ They also showed a remnant of the point node with nonzero SC gap extends along $k_{\mathrm{z}}$. Thus the ARPES experimental evidence showing sudden decrease in SC gap at point D connected with the vector I agrees well with this theoretical study. The location on FSs and $k_{\mathrm{z}}(\sim 0.375)$ of the vector II can be related to the vector B in Ref. 29 that connects two points on the calculated FS at $k_{\mathrm{z}}=0.5$ with the antiferromagnetic fluctuations having $(0.55,0,0)$, though Ref. 29 does not expect any local SC gap minima there. Recently, Kontani proposed a theory that microscopically explained a mechanism of an anisotropic $s$-wave superconductivity with deep SC gap minima by solving the strong-coupling Eliashberg equation in a system where strong electron-phonon coupling and moderate antiferromagnetic fluctuations coexist under an assumption of isotropic FS. ${ }^{30}$ According to this theory, each pair of SC gap minima is connected by the nesting vector. This may be in line with the present observation of the gap minima at the elliptical FS sheet. The fact that the gap value shows a rather flat region $\left[0.3-0.4 \mathrm{~A}^{-1}\right.$ of Fig. 3(c)] might suggest existence of an extended nesting region along the FS sheet, as is implied from Fig. 3(a). On the other hand, the clear difference in the gap anisotropy for different FS sheets suggests possible coexistence of anisotropic and isotropic SC gaps in borocarbides, investigations of which are a challenge for theoretical studies.

In conclusion, we have studied the $k$-dependent SC gap of $\mathrm{YNi}_{2} \mathrm{~B}_{2} \mathrm{C}(001)$ by using ultrahigh-resolution ARPES. The results provide experimental evidence for the anisotropic SC gap from $k$-resolved electronic structure point of view. The $k$ positions of pointlike SC gap minimum can be correlated with the known nesting vector, suggesting close correlation between node formation and the nesting vector. On the other hand, a local SC gap minimum has also been observed, which cannot be related to the known nesting vector but can be correlated with known antiferromagnetic fluctuations. The present study reveals complexity of many body interactions in a phonon-mediated superconductor, which leads to deeper understanding for anisotropic superconductivity.

We thank H. Harima, K. Machida, Y. Ichioka, and H. Kontani for very valuable discussion. We also thank $\mathrm{T}$. Aizawa for showing their system for preparing clean singlecrystal surfaces. This work was supported by Grant-in-aid from the Ministry of Education, Science, and Culture of Japan.
*Present address: Research Department, NISSAN ARC, LTD, Yokosuka, Kanagawa 237-0061, Japan.

${ }^{\dagger}$ Corresponding author; yokoya@cc.okayama-u.ac.jp

FPresent address: National Institute for Material Research, Tsukuba, Ibaraki 305-0047, Japan.

\$Present address: Department of Physics, College of Science and Technology, Nihon University, Chiyoda-ku, Tokyo 101-8308, Japan.

"Present address: Department of Physics, Okayama University, 3-1-1 Tsushima-naka, Okayama 700-8530, Japan.

${ }^{1}$ J. R. Schrieffer, Theory of Superconductivity (Perseus Books, Reading, MA, 1983).

${ }^{2}$ K. H. Bennemann and J. B. Ketterson, The Physics of Superconductors (Springer-Verlag, Berlin, 2004).

${ }^{3}$ L. F. Mattheiss, Phys. Rev. B 49, 13279 (1994); L. F. Mattheiss, T. Siegrist, and R. J. Cava, Solid State Commun. 91, 587 (1994).

${ }^{4}$ W. E. Pickett and D. J. Singh, Phys. Rev. Lett. 72, 3702 (1994).

${ }^{5}$ J. I. Lee, T. S. Zhao, I. G. Kim, B. I. Min, and S. J. Youn, Phys. Rev. B 50, 4030 (1994).

${ }^{6}$ D. D. Lawrie and J. P. Franck, Physica C 245, 159 (1995).

${ }^{7}$ K. O. Cheon, I. R. Fisher, and P. C. Canfield, Physica C 312, 35 (1999).

${ }^{8}$ M. Nohara, M. Isshiki, H. Takagi, and R. J. Cava, J. Phys. Soc. Jpn. 66, 1888 (1997).

${ }^{9}$ E. Boaknin, R. W. Hill, C. Proust, C. Lupien, L. Taillefer, and P. C. Canfield, Phys. Rev. Lett. 87, 237001 (2001).

${ }^{10}$ T. Yokoya, T. Kiss, T. Watanabe, S. Shin, M. Nohara, H. Takagi, and T. Oguchi, Phys. Rev. Lett. 85, 4952 (2000).

${ }^{11}$ G.-Q. Zheng et al., J. Phys. Chem. Solids 59, 2169 (1998).

${ }^{12}$ I.-S. Yang, M. V. Klein, S. L. Cooper, P. C. Canfield, B. K. Cho, and S. I. Lee, Phys. Rev. B 62, 1291 (2000).

${ }^{13}$ B. H. Brandow, Philos. Mag. 83, 2487 (2003).
${ }^{14}$ J. Y. Rhee, X. Wang, and B. N. Harmon, Phys. Rev. B 51, 15585 (1995).

${ }^{15}$ S. B. Dugdale, M. A. Alam, I. Wilkinson, R. J. Hughes, I. R. Fisher, P. C. Canfield, T. Jarlborg, and G. Santi, Phys. Rev. Lett. 83, 4824 (1999).

${ }^{16}$ K. Izawa, K. Kamata, Y. Nakajima, Y. Matsuda, T. Watanabe, M. Nohara, H. Takagi, P. Thalmeier, and K. Maki, Phys. Rev. Lett. 89, 137006 (2002).

${ }^{17}$ T. Watanabe, M. Nohara, T. Hanaguri, and H. Takagi, Phys. Rev. Lett. 92, 147002 (2004).

${ }^{18}$ H. Nishimori et al., J. Phys. Soc. Jpn. 73, 3247 (2004).

${ }^{19}$ T. Park, M. B. Salamon, E. M. Choi, H. J. Kim, and S. I. Lee, Phys. Rev. Lett. 90, 177001 (2003).

${ }^{20}$ C. L. Huang, J. Y. Lin, C. P. Sun, T. K. Lee, J. D. Kim, E. M. Choi, S. I. Lee, and H. D. Yang, Phys. Rev. B 73, 012502 (2006).

${ }^{21}$ S. Mukhopadhyay, G. Sheet, P. Raychaudhuri, and H. Takeya, Phys. Rev. B 72, 014545 (2005).

${ }^{22}$ T. Yokoya et al., J. Phys. Chem. Solids 67, 277 (2006).

${ }^{23}$ P. Starowicz et al., Phys. Rev. B 77, 134520 (2008).

${ }^{24}$ H. Takeya, T. Hirano, and K. Kadowaki, Physica C 256, 220 (1996).

${ }^{25}$ S. Tsuda, T. Yokoya, Y. Takano, H. Kito, A. Matsushita, F. Yin, J. Itoh, H. Harima, and S. Shin, Phys. Rev. Lett. 91, 127001 (2003).

${ }^{26}$ H. Kawano, H. Yoshizawa, H. Takeya, and K. Kadowaki, Phys. Rev. Lett. 77, 4628 (1996).

${ }^{27}$ T. Kohara et al., Phys. Rev. B 51, 3985 (1995).

${ }^{28}$ K. Ikushima et al., J. Phys. Soc. Jpn. 63, 2878 (1994).

${ }^{29}$ Y. Nagai, Y. Kato, N. Hayashi, K. Yamauchi, and H. Harima, Phys. Rev. B 76, 214514 (2007).

${ }^{30}$ H. Kontani, Phys. Rev. B 70, 054507 (2004). 